\title{
Ultra-robust stretchable electrode for e-skin: in situ assembly using a nanofiber scaffold and liquid metal to mimic water-to-net interaction
}

\section{Jin Wei Cao}

University of Nottingham Ningbo China https://orcid.org/0000-0001-9681-1155

Fei Liang

The Hong Kong Polytechnic University

Hua Yang Li

University of Nottingham Ningbo China

Xin Li

Beijing Institute of Nanoenergy and Nanosystems

You Jun Fan

Tsinghua University

\section{Yiming Yin}

University of Nottingham Ningbo China

\section{Fali Li}

CAS Key Laboratory of Magnetic Materials and Devices, Ningbo Institute of Materials Technology and Engineering

Jin Xu

University of Nottingham Ningbo China

\section{Hanfang Feng}

University of Nottingham Ningbo China

\section{Dan Xu}

CAS Key Laboratory of Magnetic Materials and Devices, Ningbo Institute of Materials Technology and Engineering, Chinese Academy of Sciences

\section{Huali Yang}

Chinese Academy of Sciences

\section{Yiwei Liu}

Ningbo Institute of Materials Technology and Engineering, Chinese Academy of Sciences

\section{Run-Wei Li}

Ningbo Institute of Industrial Technology https://orcid.org/0000-0003-3879-9834

\section{Guang Zhu ( $\nabla$ guang.zhu@nottingham.edu.cn )}

University of Nottingham Ningbo China 


\section{Article}

Keywords: electronic devices, materials science, e-skin

Posted Date: April 15th, 2021

DOl: https://doi.org/10.21203/rs.3.rs-386313/v1

License: (c) (i) This work is licensed under a Creative Commons Attribution 4.0 International License. Read Full License

Version of Record: A version of this preprint was published at InfoMat on March 5th, 2022. See the published version at https://doi.org/10.1002/inf2.12302. 


\section{Abstract}

The development of stretchable electronics will thrive on the novel interface structure to solve the stretchability-conductivity dilemma, which is still a great challenge. Herein, we report a nano-liquid metal (LM)-based high-robust stretchable electrode (NHSE) with a self-adaptable interface that mimics water-tonet interaction. Based on in situ assembly of electrospun elastic nanofibers scaffold and electrosprayed LM nanoparticles, the NHSE exhibits an extremely low sheet resistance of $52 \mathrm{~m} \Omega / \square$. It is not only insensitive to a large degree of mechanical stretching (i.e., 350\% electrical resistance change upon $570 \%$ elongation), but also immune to cyclic deformation (i.e., $5 \%$ electrical resistance increase after 100,000 stretching cycles with $100 \%$ elongation). These key properties are far more superior to the state-of-the-art reports. Its robustness and stability are verified under diverse circumstances, including long-term exposure in air (420 days), cyclic washing (30,000 times), and resilience against mechanical damages. The combination of conductivity, stretchability and durability makes the NHSE a promising conductor/electrode solution to flexible/stretchable electronics for applications such as wearable onbody physiological signal detection.

\section{Introduction}

Till now, stretchable electrodes are categorized into three major types, i.e., structure-based stretchable electrodes, intrinsically stretchable conductors, and composite-based stretchable electrodes ${ }^{10,11}$. Jang et al. prepared a three-dimensional (3D) helical microcoils layout to offer elastic mechanics for advanced components interconnection ${ }^{12}$. Wang et al. pioneered in developing poly(3,4ethylenedioxythiophene):poly(styrenesulfonate) (PEDOT:PSS) film to realize high conductivity of 4100 $\mathrm{S} / \mathrm{cm}$ under $100 \%$ stain $^{13}$. Jin et al. developed ink-permeated stretchable e-textiles that presented $7000 \%$ resistance variation under $450 \%$ elongation ${ }^{14}$. However, these stretchable electrodes are still not robust enough under high strain or cyclic stretching.

Recently, liquid metal (LM), generally non-toxic gallium-based alloys ${ }^{15}$, has emerged as a possible solution, e.g., 3D-Calabash bunch conductive network ${ }^{16}$. Although high surface tension of LM and poor interface interaction between LM and elastomers make it challenging to maintain conductivity under large strain ${ }^{17}$, two major methods have been developed to enhance the adhesion between LM and elastomers, i.e., alloying LM with other elements (AgNP-Ga-In or $\left.\mathrm{AuGa}_{2}\right)^{18,19}$, and adding binder materials to form hydrogen bonds (fructose or hydrogel) ${ }^{20,21}$. However, these methods unfortunately failed to achieve a breakthrough in the conductivity-stretchability dilemma ${ }^{22,23}$. Thus, it is still a major challenge to simultaneously achieve high stretchability, electrical stability, and cyclic durability for LM-based stretchable electrodes.

Here we demonstrate a nano-LM-based high-robust stretchable electrode (NHSE) via in situ assembly of electrospun fibrous elastic scaffolds (thermoplastic polyether urethanes, TPU) and electrosprayed LM nanoparticles. An ultra-low sheet resistance of $52 \mathrm{~m} \Omega \mathrm{sq}^{-1}$ is achieved with a standard deviation of $5 \mathrm{~m} \Omega$ 
$\mathrm{sq}^{-1}$. The NHSE presents an excellent electromechanical property with only $350 \%$ resistance variation under an elongation of $570 \%$. This outstanding property is attributed to a self-adaptable interface between the LM and the nanofibers, which mimics the water-to-net interaction. Under a small strain ( $<100 \%$ elongation), the interface stress is relaxed due to the flow of the LM, inhibiting the formation of cracks and maintaining a continuous LM film. Under a large strain $(100 \%-500 \%)$, the nanofiber scaffold serves as a local confining boundary that impedes the propagation of cracks. More importantly, the NHSE delivers superior robustness against dynamic cyclic stretching and environment stimulus (i.e., heating, acid \& alkali exposure, and washing). Specifically, it demonstrates only $5 \%$ resistance variation after 100,000 stretching cycles at a $100 \%$ cyclic strain, which shows superior robustness to other recently reported electrodes. Using the ultrathin and highly robust NHSE, we successfully fabricated wearable devices for long-term ECG monitoring and electrically self-healing connector, which contributes to the future development of innovative E-skins for biosensing and in vivo bio-stimulation.

\section{Results}

\section{The preparation of the NHSE and its static electrical performance}

The fabrication process of the NHSE is presented in Figure 1a. The TPU nanofibers were electrospun from TPU-hexafluoroisopropanol (HFIP) solution, which generated fine ( $\sim 00 \mathrm{~nm}$ in diameter, Supplementary Fig. 1) and robust nanofibers ${ }^{24}$. The LM nanoparticles were dispersed in ice-bathed isopropanol solution that enabled stable LM nanoparticle suspension ${ }^{25}$ to facilitate subsequent mechanical activation process (Supplementary Fig. 2). Then, the TPU nanofibers and the LM nanoparticles were collected on a metal collector simultaneously by electrospinning and electrospraying, respectively [Fig. $1 \mathrm{a}(\mathrm{i})$ ]. After this process, the LM nanoparticles were bonded onto the TPU nanofibers through electrostatic force to form a LM nanoparticles @ TPU scaffold composite (LNSC) [Fig. 1a(ii)] ${ }^{26}$. As revealed by the top-down view of scanning electron microscopic (SEM) (Fig. 1b), the LM nanoparticles were composed of nano-sized particles and micro-sized ones that might be agglomerated from smaller ones. These LM nanoparticles were densely adhered on the TPU nanofibers scaffold. To break the oxide shell of the LM nanoparticles and achieve a conductive pathway, an external stimulus, e.g., scraping, was conducted on top of the specimen for the coalescence of the LM nanoparticles. Consequently, an ultrathin highly conductive LM film on the top part was formed, as sketched in Figure 1a(iii). The LM film showed a continuous morphology (Fig. 1C), which is supported by the porous nanofibers-based scaffold underneath. This phenomenon is due to the balance between the Laplace capillary force $\left(F_{1}\right)$ and the gravitational force $(\mathrm{Fg})$, as illustrated in Supplementary Figure 3. It has the same nature as the water-to-net interaction in the macroscopic world. The overall thickness of the NHSE is $50 \mu \mathrm{m}$, including an ultrathin LM membrane with a thickness of hundreds of nanometres on the top (Fig. 1d). It is possible that the LM nanoparticles fabrication lessened the surface tension of LM to enable such a LM membrane that is thinner than other works $21,27$. 
The NHSE exhibits an excellent intrinsic electrical performance, as illustrated in Figure 1e, f. Specifically, the sheet resistance of the NHSE reaches as low as $52 \mathrm{m \Omega} \mathrm{sq}{ }^{-1}$ under an electrospraying rate of 0.08 $\mathrm{ml} / \mathrm{min}$. The dependence of the sheet resistance on the electrospraying rate is presented in Figure $1 \mathrm{e}$. The sheet resistance becomes lower due to the larger quantity of LM with increasing spraying speed (Supplementary Fig. $4 \mathrm{a}-\mathrm{d}$ ). Based on the mapping of the sheet resistance in an area of $2 \times 2 \mathrm{~cm}^{2}$, a standard deviation of $5 \mathrm{~m} \Omega \mathrm{sq}^{-1}$ was obtained. (Fig. 1f). As the TPU scaffold enables exceptional mechanical stretchability and mechanical compliance, the NHSE shows a stretchability with a uniaxial elongation of $\sim 570 \%$ (Fig. 1g). Illustrated in Figure 1h, it forms a seamless and conformal contact on human forearm without adhesives. No exfoliation was observed upon dynamic deformation of the skin. Besides, it is worth noting that the fabrication process is compatible to possible area-scalable production, as demonstrated by a sample with dimensions of $10 \mathrm{~cm} \times 30 \mathrm{~cm}$ (Fig. 1i).

\section{Electrical characterization of the NHSE under mechanical and environmental stimuli}

The stability and robustness under mechanical deformation are the most critical criteria for stretchable electrodes, which were the highlights of recent works ${ }^{28,29}$. The superior performance of the NHSE lies in three aspects. First, its electrical resistance is insensitive to large mechanical stretching; Second, the electrical resistance keeps stable despite of a large number of cyclic deformations; Third, it shows a high level of robustness against diverse environmental conditions, e.g., fluid soaking with different $\mathrm{pH}$ values, repeated washing, temperature variation, and long-time air exposure. The detailed results are presented below.

Frist, the electrical resistance was monitored as a uniaxial stretching was applied. For the sample made with an electrospraying speed of $0.08 \mathrm{ml} / \mathrm{min}$, the resistance increases only by $17 \%$ and $350 \%$ at an elongation of $100 \%$ and $570 \%$, respectively (Fig. 2a). In comparison, the resistance variation was found to be $1,000-8,000 \%$ at an elongation range of $300-600 \%$ in very recent state-of-the-art works ${ }^{1,14,29,30}$. Besides, the resistance can almost immediately recover to its original value once the stretching is released. For example, if a sample undergoes four stretching cycles as follows, i.e., $0 \% \rightarrow 100 \% \rightarrow 0 \% \rightarrow$ $200 \% \rightarrow 0 \% \rightarrow 300 \% \rightarrow 0 \% \rightarrow 400 \% \rightarrow 0 \%$ (Fig. $2 \mathrm{~b}$ ). There are only $14 \%(26 \mathrm{~m} \Omega)$ and $48 \%(270 \mathrm{~m} \Omega)$ resistance variations after the first and the fourth cycles, respectively. Figure $2 \mathrm{c}$ exhibits the sheet resistance versus the mechanical stretchability. Having a combination of stretchability and conductivity,

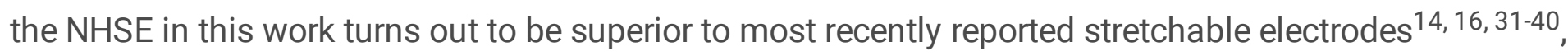
including LM-elastomer composite, Fe-LM, Ag-LM, and Au-LM composite, Ag flakes, Ag NWs, conductive polymer, etc.

Second, the NHSE possesses exceptional electrical stability under dynamic cyclic deformations, illustrated in Figure 2d, e. After being stretched with a $100 \%$ elongation for 100,000 cycles at $0.2 \mathrm{~Hz}$, the intrinsic electrical resistance without strain increases only by $5 \%$ (Fig. 2d). The slight increase is possibly due to the formation of the micro-scale wrinkles on the NHSE (Supplementary Fig. 5a-c), which induces a prolonged conductive pathway. It is interesting that the resistance variation becomes smaller as more 
stretching cycles are applied (Fig. 2d insets). This can be also explained by the micro-wrinkles, which has been known for mitigating effective strain applied on stretchable devices ${ }^{41,42}$.

Third, the NHSE almost constantly maintains the resistance as being stretched at $0 \%, 100 \%$ or $200 \%$ under different environmental conditions (Fig. 2e and Supplementary Fig. 6, 7). The resistance of the stretched NHSE barely changes after 1,000 washing cycles (Supplementary Fig. 6). There is a merely $11 \%$ resistance rise for a $200 \%$-stretched NHSE after 30,000 washing cycles (Fig. 2e). Besides, samples soaked in artificial perspirations with $\mathrm{pH} 4.7$ and 8.8 present a negligible resistance increase, respectively (Supplementary Fig. 7a, b). As for long-term stability of the NHSE in ambient environment, the sheet resistance remains approximately $60 \mathrm{~m} \Omega \mathrm{sq}^{-1}$ with a negligible variation after being exposure in air after 420 days (Fig. 2f). Also, the resistance stability of the NHSE was investigated through freezing and heating processes (Fig. $2 \mathrm{f}$ inset). The resistance of the NHSE gradually increased by $50 \%$ as the sample temperature varied from $-30^{\circ} \mathrm{C}$ to $120^{\circ} \mathrm{C}$. The increase possibly comes from the oxidation of the $\mathrm{LM}$ surface at the elevated temperature. It is worth noting that there is a sharp increase of the electrical resistance at approximately $15{ }^{\circ} \mathrm{C}$, at which a phase transition of the LM from solid to liquid probably occurs ${ }^{43,44}$.

\section{Mechanism of the electrical robustness of the NHSE}

Nature always enlightens us through various wonderful workmanship in materials design and mechanism analysis ${ }^{45}$. The NHSE highly resembles a natural phenomenon, in which a continuous water membrane spreads across a meshed fishing net (Fig. 3a). The evolution of the interaction between the water membrane and the meshed net is illustrated in Supplementary Fig. 8a-c as a strain is applied. The water membrane is deformable and maintains continuity at the initial stage of the strain. Under a large strain, if even cracks are generated, they are confined within individual mesh units without causing total rupture of the water membrane.

In the case of the NHSE, similar observations were found. To reveal the morphology evolution, samples with different levels of strain were examined under SEM (Fig. 3b-j). Here, the interface between the LM and the nanofibers is adaptable upon deformation, which is the key for the high level of electrical stability of the NHSE. This adaptable behaviour is presented in two stages, as sketched in Fig. 3b. The first stage involves a small strain range when the elongation is less $100 \%$. In this stage, the morphology of the LM membrane evolves along with the deformation of the nanofibers without interface stress being built up. As a result, the LM membrane remains integral, which is justified by the SEM image as well as a video with in-situ stretching of $100 \%$ elongation (Fig. 3c, d and Supplementary Movie 1). Although random minor pits could be identified (Fig. 3d), neither proliferation nor propagation of the pits were found in subsequent stretching cycles (Fig. 3e, f). In the second stage in which the elongation increases beyond $100 \%$, more pits are generated, and they expand to micro-scale voids at the same time. However, the voids do not propagate throughout the entire LM membrane. Instead, they are confined locally by the nanofibers that act as a boundary for isolating the voids from surrounding area (Fig. $3 \mathrm{~g}, \mathrm{~h}$ ). This confinement effect prevents the LM membrane from total rupture and preserves active conducting 
pathways. Even if the elongation reaches $500 \%$, the confinement is still effective despite of largely expanded and distorted voids. As a result, the NSHE remains to be functional. The distortion observed in Figure $3 i, j$ is due to the re-orientation of the nanofibers under a uniaxial strain. The alignment of the nanofibers is quantitatively characterized by SEM images and the 2D Fast Fourier Transform (FFT) plots in Supplementary Figure 9, $10^{46}$.

Based on the above analysis, the self-adaptable interfacial interaction is the key enabler for the insensitivity as well as the robustness of the electrical resistance against strain. To obtain this particular interface, the in-situ assembly fabrication method in this work plays a vital and irreplaceable role. In order to further prove this point, control experiments with other typical fabrication methods were conducted. In the first group, LM droplets were applied onto a commercial TPU substrate, and were scratched across the substrate to form a continuous film subsequently (Supplementary Movie 2). Without the selfadaptable interface, the LM film is broken into separated islands under an elongation of $100 \%$, which is an irreversible failure (Supplementary Fig. 11). In the second group, a TPU-nanofiber-based scaffold was used as a substrate, and LM droplets were subsequently applied via the same procedure in the first group. This fabrication method was also reported in a very recent work ${ }^{27}$. The reported results showed that the electrical resistance of the stretchable electrode increased by $30 \%$ after 25,000 stretching cycles $(100 \%$ elongation). In contrast, the NHSE in this work experienced only a $5 \%$ increase in the electrical resistance after 100,000 stretching cycles.

\section{The application of the NHSE for stretchable conductor and ECG recordings}

The NHSE shows a great promise of being used as interconnects in elastic electronic systems. As a demonstration, it was applied as an elastic conductor to power a stripe of light emitting diodes ( 75 LEDs), presented in Figure 4a, b. It was stretched from original dimensions to an elongation of approximately $500 \%$. Negligible variation of the LED's brightness was observed. The repeatability was demonstrated through multiple stretching cycles. Still, the LED's brightness remained constant (Supplementary Movie 3).

Being ultrathin and mechanically compliant, the NHSE is also suitable to be used in epidermal devices for on-body physiological signal collection (Fig. $4 \mathrm{c}-\mathrm{i}$ and Supplementary Movie 4). In the following demonstration, the NHSE formed a tight and conformal bonding on human skin via van der Waals force ${ }^{47}$. Used as an epidermal electrode for electrocardiogram (ECG) recording, it has a comparable contact impedance as a commercial AgCl gel electrode (Fig. 4c). At a static state, the signal obtained from the NHSE is equivalently recognizable as that from the commercial gel electrode (Fig. 4d). The key advantage of the NHSE for this application lies in stable signal detection under external disturbance. Shown in Fig. 4f, the signal recording was accompanied by wrist movement. As a result, baseline fluctuation was found for the gel electrode, while the signal from the NHSE was unchanged. If water was poured onto the gel electrode during signal recording, significant noise buried the signal without recognizable regular patterns (Fig. 4i). In contrast, key features from the NHSE, i.e., P-waves, QRS 
complex, and T-waves, were still preserved and remained diagnosable. This contrast is attributed to seamless and conformal on-body contact from the NHSE.

\section{The electrical self-healing property of the NHSE}

The robustness of the NHSE is further verified as it survives from severe damages, including cutting and hole punching. As demonstrated in Figure 5a, a piece of NHSE was selectively and locally activated by drawing a line on top of the sample. This line became conductive and was used as a flexible circuit to power a LED. The conductive line was cut through using a razor blade on the left side of the LED.

Subsequently, a hole punching was conducted on the other side of the LED. During and after the above operation, the LED was still lighting (Fig. 5a). The damaged flexible circuit was still functional even if it was stretched afterwards (Fig. 5b).

This observation is due to the reconstruction of conductive pathways at the damaged sites. As illustrated in Figure $5 \mathrm{c}$, the line in the middle of the sample represents the region where the activation has taken place. For the rest of the area, it is subject to subsequent activation upon mechanical stimuli. Although cutting or punching disconnects the original conductive pathway, it essentially activates new sites around the cutting/punching mark (Fig. 5d, f). As a result, a fresh conductive pathway is reconstructed at the same time when the damage happens, which enables uninterrupted power supply. Quantitatively revealed in Figure $5 \mathrm{~g}$, a five-fold increase of the electrical resistance is observed after the first cut, and another $400 \%$ and $600 \%$ increase after the second and the third cuts, respectively. Similar observations were also found in the case of successive hole punching. Therefore, the NHSE shows an exceptional level of robustness despite of severe damages.

\section{Discussion}

We have proposed a nano-LM-based high-robust stretchable electrode based on liquid metal nanoparticles and TPU nanofiber scaffold by mimicking the water-to-net interface for long-term dynamic human healthcare monitoring and electrically self-healing stretchable connector. Without alloying or adding binder materials, the as-prepared NHSE realizes a self-adaptable interface, through which the LM membrane remains continuity under a strain range below $100 \%$ and the LM voids are confined locally by nanofibers under larger strain till 500\%. So, this self-adaptable interfacial interaction realizes an unprecedented combination of super low resistance under high elongation and exceptional electrical robustness upon cyclic external stimulus. These are essentials characters that contribute to the all-day on-body ECG monitoring under three scenarios, including staying static, doing exercise, and taking a shower. In addition, the robustness of circuits is further evidenced through the character of the autonomously conductive pathways' reconstruction after severe mechanical damages. This electrically self-healing process is completed spontaneously without any other manual repair or external heat. All of these demonstrate its promising potential in the application of long-term reliable E-skin or in vivo biocompatible components, e.g., brain-machine interface, neural interface, wound healing accelerator, which will be focused by the next stage investigation. 


\section{Methods}

\section{Materials for composite}

(1) EGaln eutectic alloy was fabricated through mixing high purity metal gallium (99.99\%; Beijing Founde Star Sci. \& Technol. Co., Ltd) and indium (99.995\%; Beijing Founde Star Sci. \& Technol. Co., Ltd) with the ratio of $75.5 \mathrm{wt}$. \% and \% $24.5 \mathrm{wt}$. \%, respectively. (2) The thermoplastic polyurethane (TPU) solution was prepared by dissolving TPU (Sigma-Aldrich) in a Hexafluoroisopropanol (HFIP, Aladdin) at a weight percentage of $4 \%$, then stirred for $8 \mathrm{~h}$ at room temperature.

\section{Preparation of the NHSE}

The EGaln suspension was prepared by sonicating $2.25 \mathrm{~g}$ of bulk EGaln eutectic alloy in 12.75 of isopropanol for $10 \mathrm{~min}$ in a $20 \mathrm{ml}$ vial, conducted in ultrasonic cell disruption system at $50 \%$ amplitude (Ymnl-1000Y, Nanjing Immanuel Instrument Equipment co.). The vital was placed in ice bath in this process in order to reduce solvent evaporation. Subsequently, liquid metal slurry, separated from the lighting suspension after standing for $20 \mathrm{~min}$, was transferred into a $5 \mathrm{ml}$ syringe with $19 \mathrm{G}$ metallic nozzle (Bodunbaili Co., Ltd). Besides, the homogeneous TPU solution was transferred into a plastic syringe equipped with $26 \mathrm{G}$ metallic nozzle. The EGaln and TPU solution syringes were fit on the syringe pump for electrospraying and electrospinning on electrospinning device (Beijing Tech Nova TEADFS103), respectively. The TPU nanofiber was produced with a positive voltage of $9 \mathrm{kV}$ and the EGaln slurry was sprayed uniformly on TPU nanofiber with a positive voltage of $11 \mathrm{kV}$, which were both collected on a revolving roller wrapped by aluminium foil with negative voltage of $2 \mathrm{kV}$. The needle-collector distance was $15 \mathrm{~cm}$ and $10 \mathrm{~cm}$ for TPU and EGaln, respectively. Then pump rate for TPU electrospinning and EGaln electrospraying was adjusted to be $0.04 \mathrm{ml} / \mathrm{min}$ and $0.02-0.08 \mathrm{ml} / \mathrm{min}$, respectively. Then the asfabricated LM nanoparticles @ TPU scaffold composite (LNSC) film was transferred to a $100 \mu \mathrm{m}$ PET film for further device fabrication and properties characterization.

\section{Activation of electrical circuit and electrode}

The LNSC was activated through drawing a line with a rubber tip to form a circuit, and through scraping with a PET patch to fabricate a plane electrode. The pressure applied on the sample induces the rupturing of oxide shells on LM nanoparticles and the formation of circuit or electrode mechanically.

\section{Microstructure characterization}

The surface and cross-sectional microstructures of the NHSE were characterized by the field-emission scanning electron microscopy (SEM Sirion, FEI). And the distribution of elements ( $G a$ and In) is identified by the energy-dispersive X-ray spectrum (EDS, GENESIS).

\section{Electrical Characterization}


(1) Sheet resistance: The sheet resistance of the NHSE with different electrospraying speed was measured by a four-probe resistivity measurement system (Guangzhou 4-probe Tech Co. Ltd, RTS-9). (2) Electric resistance: $1 \mathrm{~cm} \times 1 \mathrm{~cm}$ samples were prepared for electric resistance measurement in single stretching and cyclic stretching. $2 \mathrm{~cm} \times 1 \mathrm{~cm}$ patches were fabricated for electric resistance measurement in bending. $3 \mathrm{~cm} \times 1 \mathrm{~cm}$ patches on $100 \mu \mathrm{m}$ thick PDMS film were fabricated for electric resistance measurement in twisting. Conductive copper wire was pasted on the ends of each sample using silver paste. The electrical resistance of the NHSE was monitored by a digital multimeter (Keithley 2611B) using a standard four-point method; (3) Contact impedance measurement: two NHSE patches $(16 \mathrm{~mm}$ in diameter) were prepared and attached onto the forearms wetted by DI water. The centre-to-centre distance of two NHSE patches maintained $5 \mathrm{~cm}$. The electrode-skin contact impedance was measured by electrochemical analyser (CHI660E) over a frequency range from 1 to $10^{5} \mathrm{~Hz}$. As for the control group, gel electrode $(\mathrm{Ag} / \mathrm{AgCl})$ from medical health care system (Heal Force PC-3000) was applied, all the experimental parameters above were unchanged.

\section{ECG measurement}

Two NHSE patches (16 $\mathrm{mm}$ in diameter with a $3 \mathrm{~cm}$ tail) were prepared and attached onto each forearm, and another one (16 $\mathrm{mm}$ in diameter with a tail) NHSE patch was pasted at $2 \mathrm{~cm}$ above the ankle of the left leg. Then, the conductive textile (lead wire) was pasted onto the tail of the NHSE with the assistant of the silver paste and a tape (3M) that maintain good connection between lead wire and patches electrically and geometrically. After that, the lead wires were connected to a commercial health monitoring machine (Heal Force PC-3000) for ECG measurement. As for the control group, gel electrodes $(\mathrm{Ag} / \mathrm{AgCl})$ were applied in ECG monitoring, and all the experimental parameters above were unchanged.

\section{Declarations}

\section{Data availability}

All data needed to evaluate the conclusions in the paper are present in the paper and/or the Supplementary Materials. Additional data related to this paper may be requested from the corresponding authors.

\section{Acknowledgements}

This research was supported by Ningbo Municipal 3315 Talent Scheme and Ningbo Scientific and Technological Innovation 2025 Major Project (Grant No. 2018B10057) by Ningbo Science and Technology Bureau, the Zhejiang Provincial Natural Science Foundation of China (Grant No. LR19F010001), the National Key R \& D Project from Ministry of Science and Technology, China (Grant Nos. 2016YFA0202703), National Science Foundation of China (Grant Nos. 51525103, 51701231 and 51931011).

\section{Author contributions}


J.C. and G.Z. conceived the idea and designed the experiment. Y.L., R.L. and G.Z supervised the research process. J.C. and F.L. designed and fabricated the NHSE. X.L. and Y.F. guide the data analysis and interpretation. J.C., Y.Y, J.X., H.F. performed the material characterization. J.C., F.L.L. carried out the related electrical characterization. J.C., H.L. and G.Z. develop the interpretation mechanism. J.C. prepared the manuscript and F.L, H.L., D.X., H.Y., R.L., G.Z., participated in the manuscript revision.

\section{Competing Interests}

The authors declare that they have no competing interests.

\section{References}

1. Matsuhisa, N. et al. Printable elastic conductors by in situ formation of silver nanoparticles from silver flakes. Nat. Mater. 16, 834-840 (2017).

2. Choi, S. et al. Highly conductive, stretchable and biocompatible Ag-Au core-sheath nanowire composite for wearable and implantable bioelectronics. Nat. Nanotechnol. 13, 1048-1056 (2018).

3. Liu, J. et al. Intrinsically stretchable electrode array enabled in vivo electrophysiological mapping of atrial fibrillation at cellular resolution. Proc. Natl. Acad. Sci. U. S. A. 117, 14769-14778 (2020).

4. Kang, S. K. et al. Bioresorbable silicon electronic sensors for the brain. Nature 530, 71-76 (2016).

5. Minev, I. R. et al. Electronic dura mater for long-term multimodal neural interfaces. Science 347, 159163 (2015).

6. Markvicka, E. J., Bartlett, M. D., Huang, X. \& Majidi, C. An autonomously electrically self-healing liquid metal-elastomer composite for robust soft-matter robotics and electronics. Nat. Mater. 17, 618-624 (2018).

7. Jiang, Z. et al. Highly Stretchable Metallic Nanowire Networks Reinforced by the Underlying Randomly Distributed Elastic Polymer Nanofibers via Interfacial Adhesion Improvement. Adv. Mater. 31, 1903446 (2019).

8. Miyamoto, A. et al. Inflammation-free, gas-permeable, lightweight, stretchable on-skin electronics with nanomeshes. Nat. Nanotechnol. 12, 907-913 (2017).

9. Norton, J. J. S. et al. Soft, curved electrode systems capable of integration on the auricle as a persistent brain-computer interface. Proc. Natl. Acad. Sci. U.S.A. 112, 3920-3925 (2015).

10. Matsuhisa, N., Chen, X., Bao, Z. \& Someya, T. Materials and structural designs of stretchable conductors. Chem. Soc. Rev. 48, 2946-2966 (2019).

11. Dickey, M. D. Stretchable and Soft Electronics using Liquid Metals. Adv. Mater. 29, (2017).

12. Jang, K. I. et al. Self-assembled three dimensional network designs for soft electronics. Nat. Commun. 8, 15894 (2017).

13. Wang, Y. et al. A highly stretchable, transparent, and conductive polymer. Sci. Adv. 3, 1602076 (2017).

14. Jin, H. et al. Enhancing the Performance of Stretchable Conductors for E-Textiles by Controlled Ink Permeation. Adv. Mater. 29, 1605848 (2017). 
15. Daeneke, T. et al. Liquid metals: fundamentals and applications in chemistry. Chem. Soc. Rev. 47, 4073-4111 (2018).

16. Yu, Z. et al. A Composite Elastic Conductor with High Dynamic Stability Based on 3D-Calabash Bunch Conductive Network Structure for Wearable Devices. Adv. Electron. Mater. 4, 1800137 (2018).

17. Ma, B. et al. A Versatile Approach for Direct Patterning of Liquid Metal Using Magnetic Field. Adv. Funct. Mater. 29, 1901370 (2019).

18. Mahmoud Tavakoli et al. EGaln-Assisted Room-Temperature Sintering of Silver Nanoparticles for Stretchable, Inkjet-Printed, Thin-Film Electronics. Adv. Mater. 30, 1801852 (2018).

19. Hirsch, A., Michaud, H. O., Gerratt, A. P., de Mulatier, S. \& Lacour, S. P. Intrinsically Stretchable Biphasic (Solid-Liquid) Thin Metal Films. Adv. Mater. 28, 4507-4512 (2016).

20. Guo, R., Sun, X., Yuan, B., Wang, H. \& Liu, J. Magnetic Liquid Metal (Fe-EGaln) Based Multifunctional Electronics for Remote Self-Healing Materials, Degradable Electronics, and Thermal Transfer Printing. Adv. Sci. 6, 1901478 (2019).

21. Park, J. E., Kang, H. S., Koo, M. \& Park, C. Autonomous Surface Reconciliation of a Liquid-Metal Conductor Micropatterned on a Deformable Hydrogel. Adv. Mater. 32, 2002178 (2020).

22. Sekitani, T. et al. Stretchable active-matrix organic light-emitting diode display using printable elastic conductors. Nat. Mater. 8, 494-499 (2009).

23. Shi, G. et al. Highly sensitive, wearable, durable strain sensors and stretchable conductors using graphene/silicon rubber composites. Adv. Funct. Mater. 26, 7614-7625 (2016).

24. Fan, Y. J. et al. Highly Conductive, Stretchable, and Breathable Epidermal Electrode Based on Hierarchically Interactive Nano-network. Nanoscale 12, 16053-16062 (2020).

25. Mohammed, M. G. \& Kramer, R. All-Printed Flexible and Stretchable Electronics. Adv. Mater. 29, 1604965 (2017).

26. Li, W. et al. Nanofibrous mats layer-by-layer assembled via electrospun cellulose acetate and electrosprayed chitosan for cell culture. Eur. Polym. J. 48, 1846-1853 (2012).

27. $\mathrm{Ma}$, Z. et al. Permeable superelastic liquid-metal fibre mat enables biocompatible and monolithic stretchable electronics. Nat. Mater. 1-10 (2021).

28. Liu, L. et al. Nanofiber-Reinforced Silver Nanowires Network as a Robust, Ultrathin, and Conformable Epidermal Electrode for Ambulatory Monitoring of Physiological Signals. Small 15, 1900755 (2019).

29. Park, S., Thangavel, G., Parida, K., Li, S. \& Lee, P. S. A Stretchable and Self-Healing Energy Storage Device Based on Mechanically and Electrically Restorative Liquid-Metal Particles and Carboxylated Polyurethane Composites. Adv. Mater. 31, 1805536 (2019).

30. Li, X. et al. Evaporation-induced sintering of liquid metal droplets with biological nanofibrils for flexible conductivity and responsive actuation. Nat. Commun. 10, 3514 (2019).

31. Cheng, Y., Wang, S., Wang, R., Sun, J. \& Gao, L. Copper nanowire based transparent conductive films with high stability and superior stretchability. J. Mater. Chem. C 2, 5309-5316 (2014). 
32. Lopes, P. A., Paisana, H., De Almeida, A. T., Majidi, C. \& Tavakoli, M. Hydroprinted electronics: ultrathin stretchable Ag-In-Ga E-skin for bioelectronics and human-machine interaction. ACS Appl. Mater. Interfaces 10, 38760-38768 (2018).

33. Tybrandt, K. et al. High-Density Stretchable Electrode Grids for Chronic Neural Recording. Adv. Mater. 30, 1706520 (2018).

34. Lee, P. et al. Highly stretchable and highly conductive metal electrode by very long metal nanowire percolation network. Adv. Mater. 24, 3326-3332 (2012).

35. Park, S.-M. et al. Metal nanowire percolation micro-grids embedded in elastomers for stretchable and transparent conductors. J. Mater. Chem. C 3, 8241-8247 (2015).

36. Hong, S. et al. Highly stretchable and transparent metal nanowire heater for wearable electronics applications. Adv. Mater. 27, 4744-4751 (2015).

37. Li, J. et al. Synthesizing a Healable Stretchable Transparent Conductor. ACS Appl. Mater. Interfaces 7 , 14140-14149 (2015).

38. Lee, M. S. et al. High-performance, transparent, and stretchable electrodes using graphene-metal nanowire hybrid structures. Nano Lett. 13, 2814-2821 (2013).

39. Xu, F., Wang, X., Zhu, Y. \& Zhu, Y. Wavy Ribbons of Carbon Nanotubes for Stretchable Conductors. Adv. Funct. Mater. 22, 1279-1283 (2012).

40. Hu, W. et al. Intrinsically stretchable transparent electrodes based on silver-nanowire-crosslinkedpolyacrylate composites. Nanotechnology 23, 344002 (2012).

41. Hyun, D. C. et al. Ordered Zigzag Stripes of Polymer Gel/Metal Nanoparticle Composites for Highly Stretchable Conductive Electrodes. Adv. Mater. 23, 2946-2950 (2011).

42. Wei, Y., Chen, S., Yuan, X., Wang, P. \& Liu, L. Multiscale wrinkled microstructures for piezoresistive fibers. Adv. Funct. Mater. 26, 5078-5085 (2016).

43. Lu, T., Wissman, J., Ruthika \& Majidi, C. Soft Anisotropic Conductors as Electric Vias for Ga-Based Liquid Metal Circuits. ACS Appl. Mater. Interfaces 7, 26923-26929 (2015).

44. Gozen, B. A., Tabatabai, A., Ozdoganlar, O. B. \& Majidi, C. High-density soft-matter electronics with micron-scale line width. Adv. Mater. 26, 5211-5216 (2014).

45. Khan, M. R., Trlica, C. \& Dickey, M. D. Recapillarity: Electrochemically Controlled Capillary Withdrawal of a Liquid Metal Alloy from Microchannels. Adv. Funct. Mater. 25, 671-678 (2015).

46. Ayres, C. E. et al. Measuring fiber alignment in electrospun scaffolds: a user's guide to the 2D fast Fourier transform approach. J. Biomater. Sci. Polym. Ed. 19, 603-621 (2008).

47. Kabiri Ameri, S. et al. Graphene Electronic Tattoo Sensors. ACS Nano 11, 7634-7641 (2017).

\section{Figures}




\section{a}

(i) (ii)

(iii)
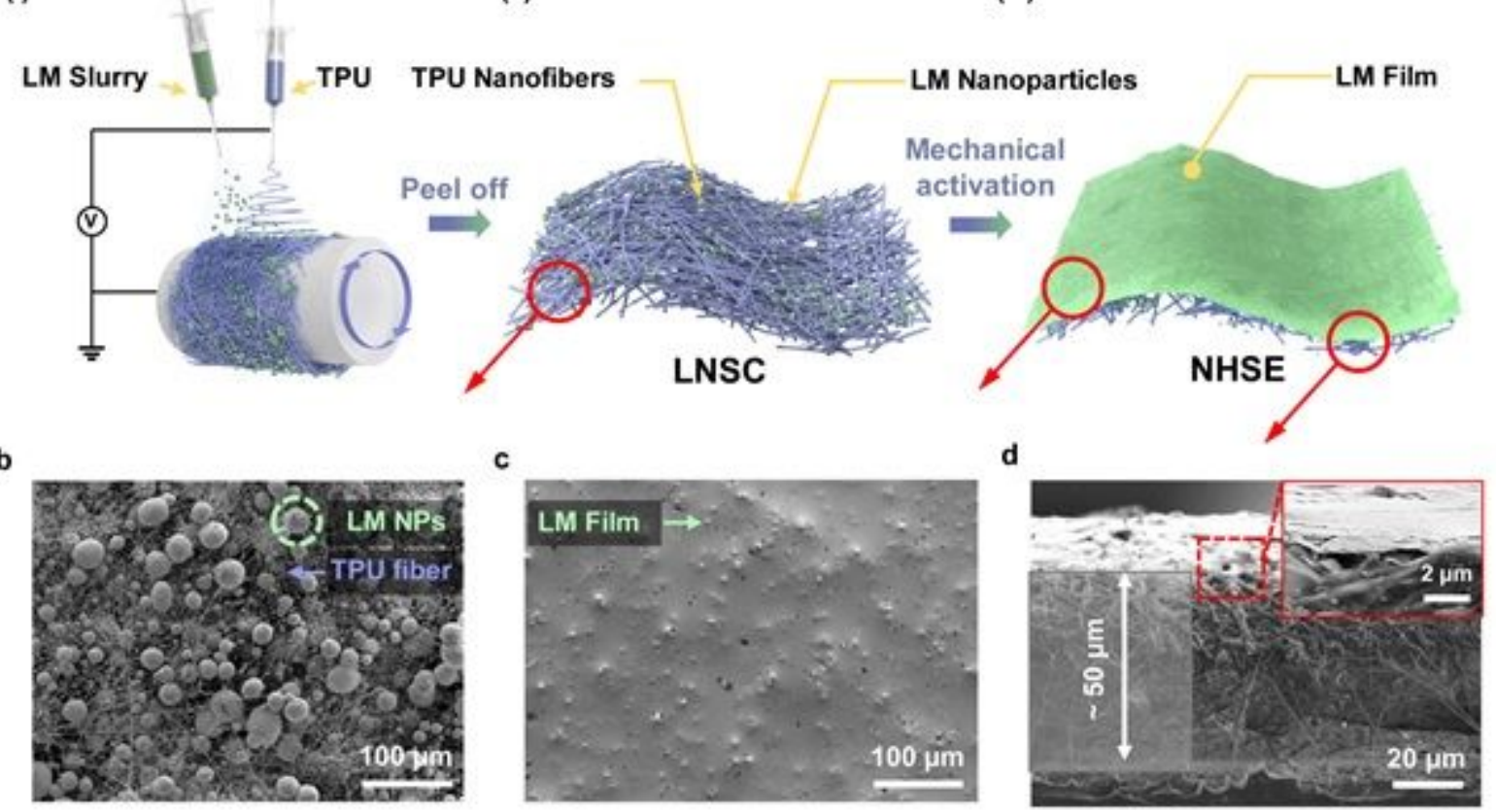

e

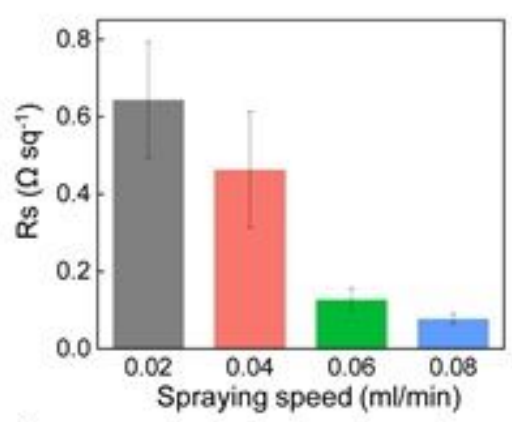

g
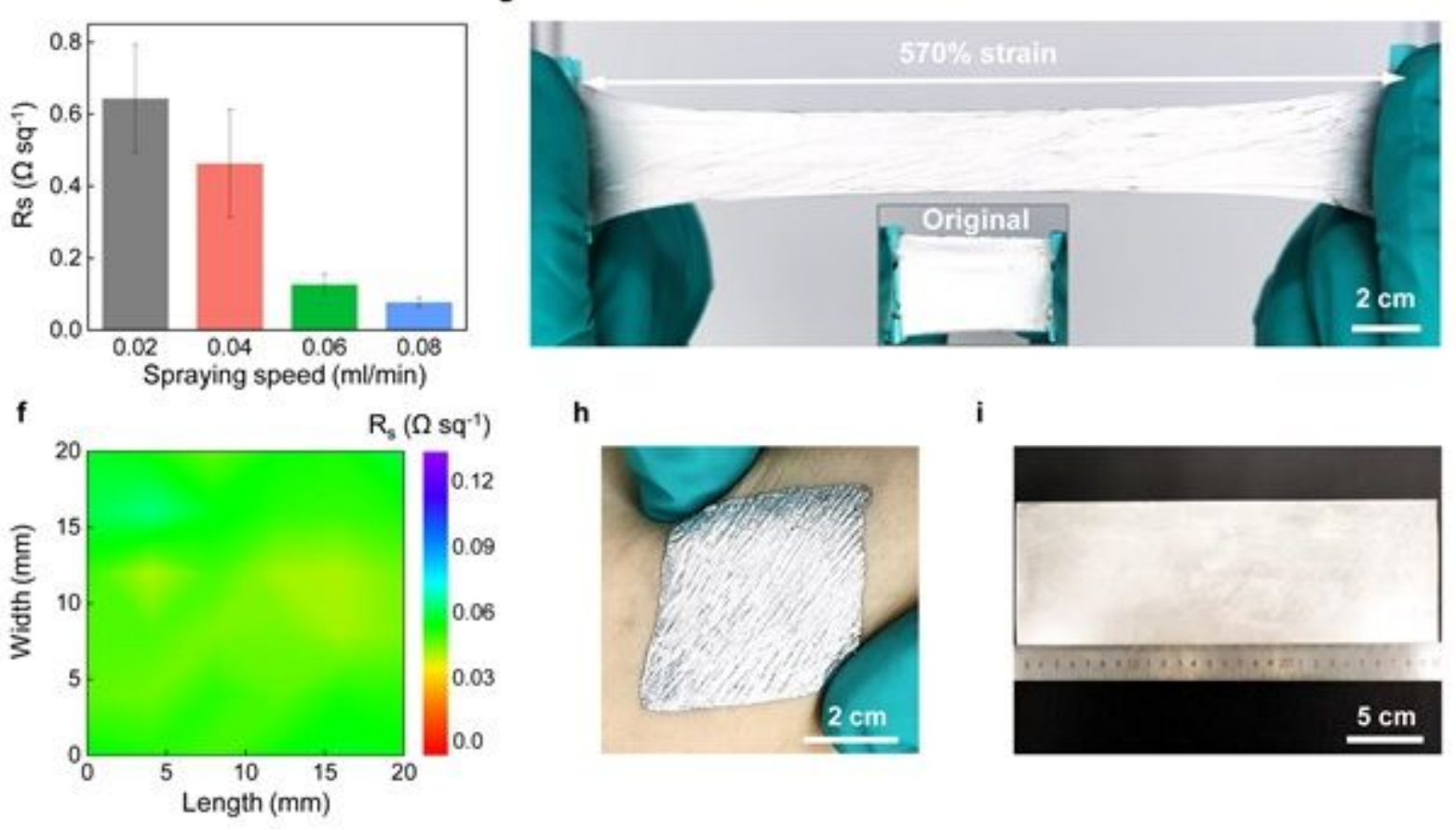

h

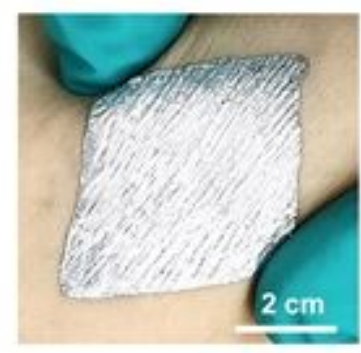

i

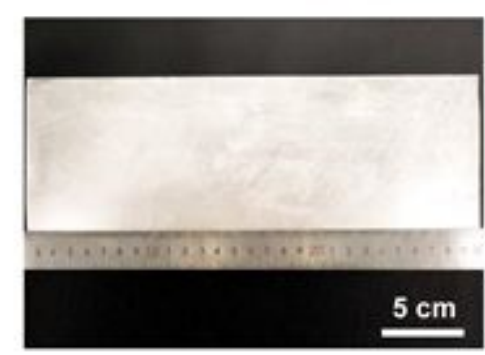

\section{Figure 1}

Design of nano-LM-based high-robust stretchable electrode (NHSE) and its static electrical performance. a Schematic illustration of the (i) electrospray and electrospinning process to fabricate (ii) the LM nanoparticles @ TPU scaffold composite (LNSC) that is subsequently activated to form (iii) the NHSE. b, c Top-down view SEM of the LNSC and the NHSE, respectively. $d$ Cross-sectional SEM image, and the inset depict the cross-section of LM membrane. e Sheet resistance of the NHSE as a function of EGaln nanoparticles solution electrospray speed. $f$ Area mapping of sheet resistance distribution of the NHSE with a size of $20 \times 20 \mathrm{~mm} 2$. g Photograph of NHSE under relaxed (inset) and $570 \%$ of elongation. $h$ 
Picture of the highly conformal NHSE attached on the forearm. i A size of $10 \mathrm{~cm} \times 30 \mathrm{~cm}$ NHSE shows its promising utility for the electrode large-scale production.
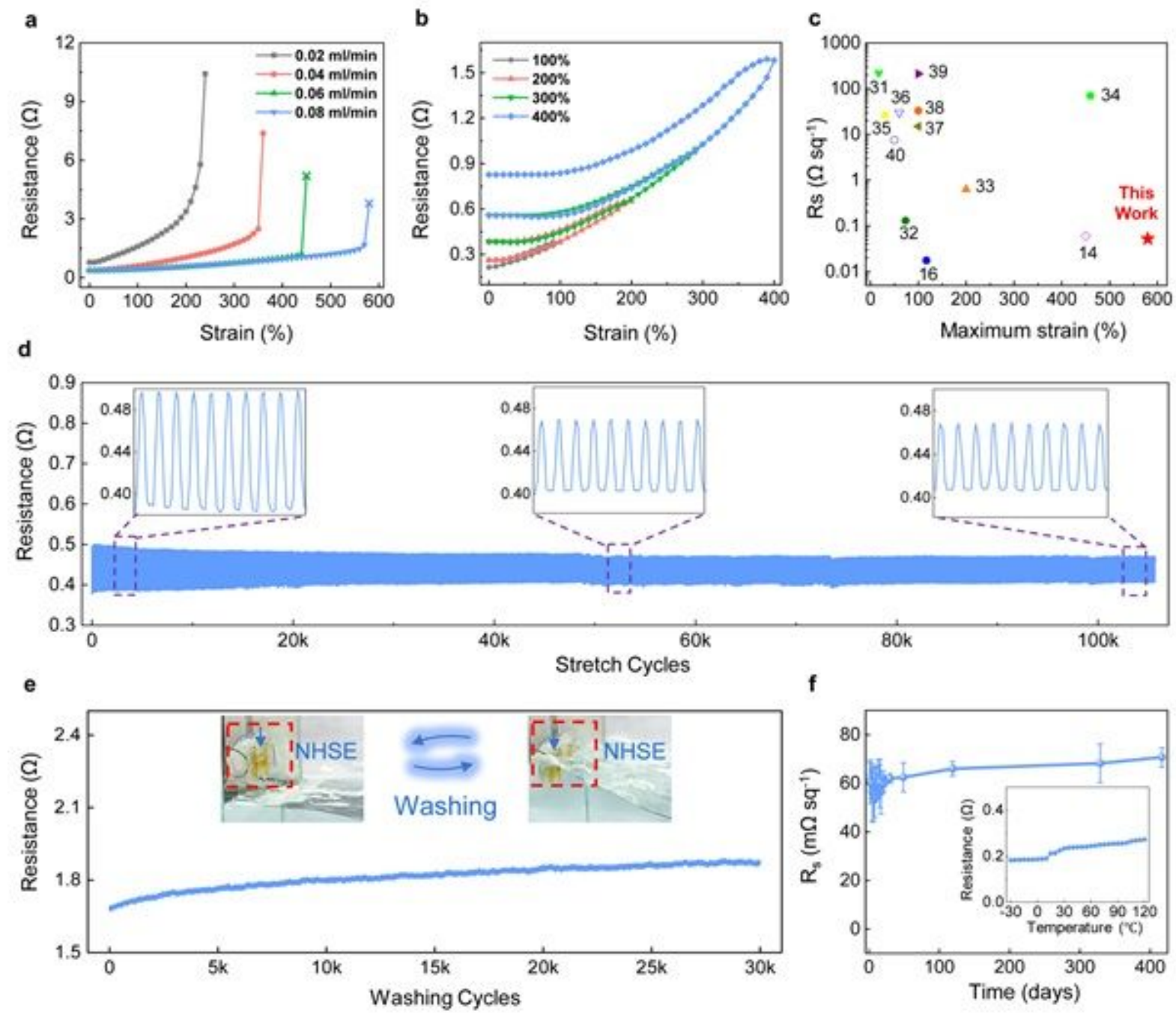

Figure 2

Electrical characterization of the NHSE under mechanical and environmental stimuli. a Resistance-strain character of the NHSE with various EGaln electros pray speed (sample size: $1 \mathrm{~cm} \times 1 \mathrm{~cm}$ ). b Resistance variation of the NHSE against stretching cycles as $0 \% \rightarrow 100 \% \rightarrow 0 \% \rightarrow 200 \% \rightarrow 0 \% \rightarrow 300 \% \rightarrow 0 \% \rightarrow$ $400 \% \rightarrow 0 \%$ (sample size: $1 \mathrm{~cm} \times 1 \mathrm{~cm}$, strain speed: $45 \% / \mathrm{min}$ ). c Comparison of the initial sheet resistance and stretchability with previusly published electrodes. $d$ Resistance of the NHSE that lasted for $\sim 100,000$ stretching under $100 \%$ strain $(0.2 \mathrm{~Hz}$, sample size: $1 \mathrm{~cm} \times 1 \mathrm{~cm})$. The insets shows the detailed resistance values at the beginning, in the middle and at the end of the cyclic tensile test. e The resistance of the $300 \%$-stretched NHSE during 30, 000 water washing cycles (initiral sample size: $1 \mathrm{~cm} \times 1 \mathrm{~cm}$ ). $f$ Sheet resistance of the NHSE as exposed in the air for 420 days, and the inset shows the resistance variation of the NHSE under an increasing temperature from $-30{ }^{\circ} \mathrm{C}$ to $120^{\circ} \mathrm{C}$. 
a

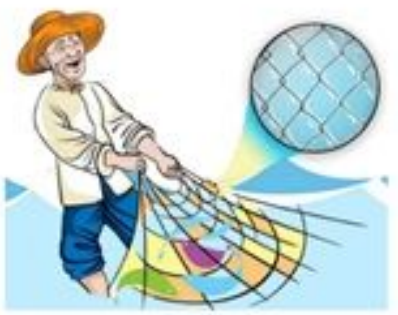

b (i)

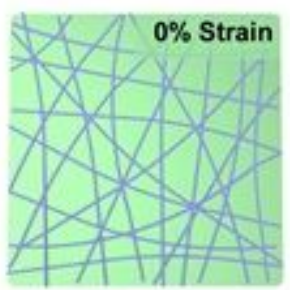

(ii)

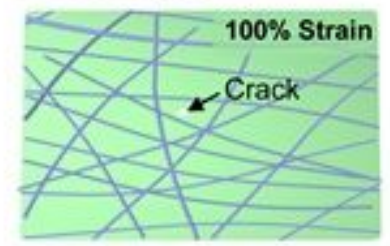

(iii)

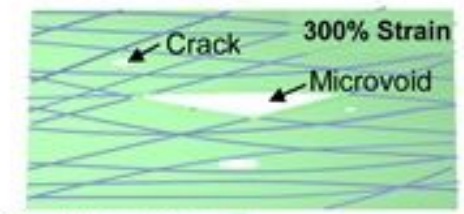

(iv) Stretching

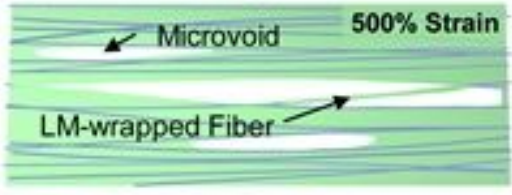

LM film c

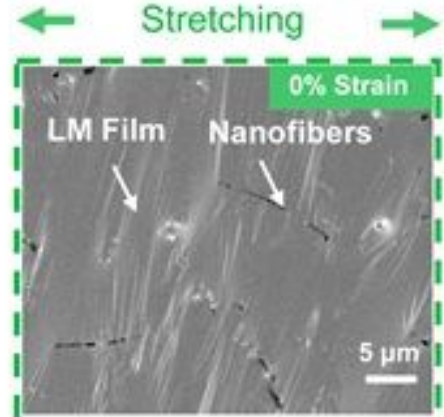

d
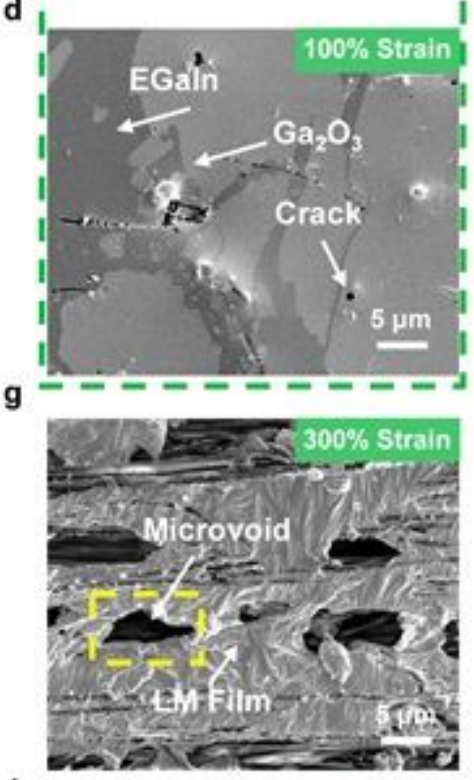

i

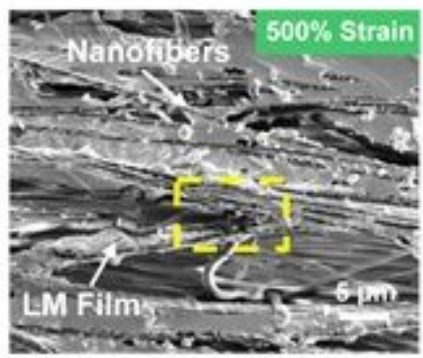

$4-$ In situ stretching ${ }^{3 r d} \Rightarrow$

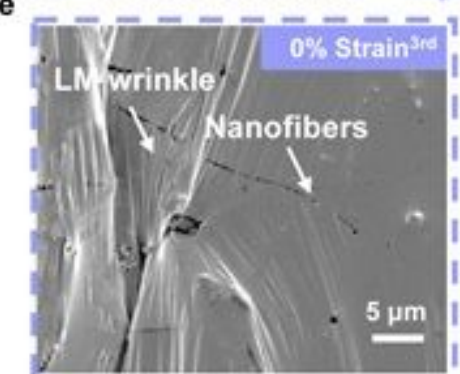

f
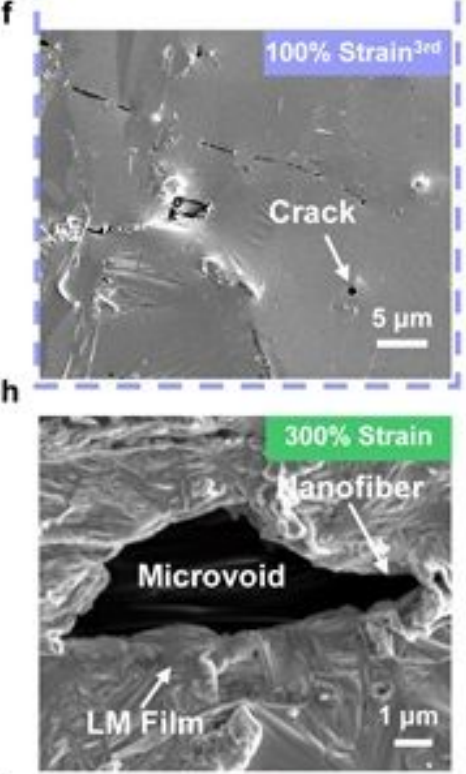

j

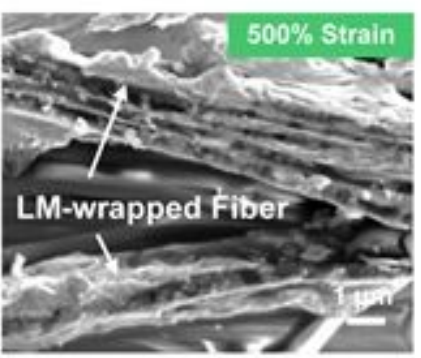

Figure 3

The self-adaptable interface between the LM membrane and TPU scaffold. a The zoom-in morphology of water membrane when fishman pulls the net from the sea. $b$ The self-adaptable interface evolution as stretched to $100 \%, 300 \%, 500 \%$ strain, respectively. $c-f$ In situ micromorphology analysis of the NHSE under 1 st 100\% strain stretching (c, d) and 3rd 100\% strain stretching (e, f). g, h Micromorphology of the NHSE under $300 \%$ elongation. i, j Micromorphology of the NHSE under $500 \%$ elongation. 
a

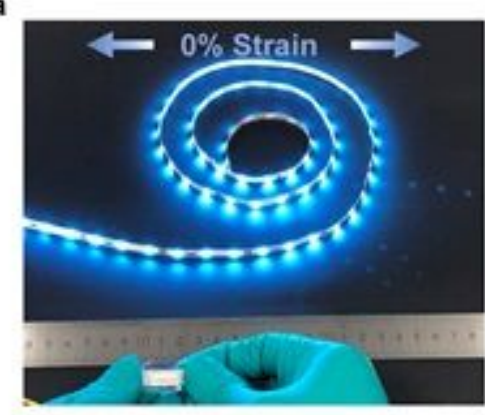

d

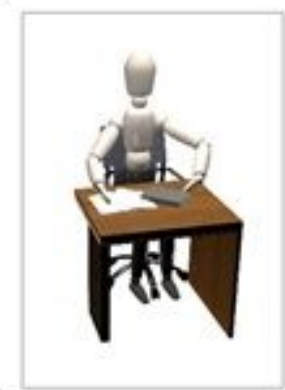

f

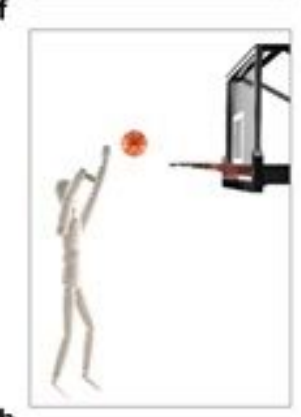

h

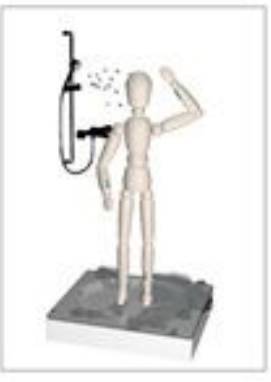

b
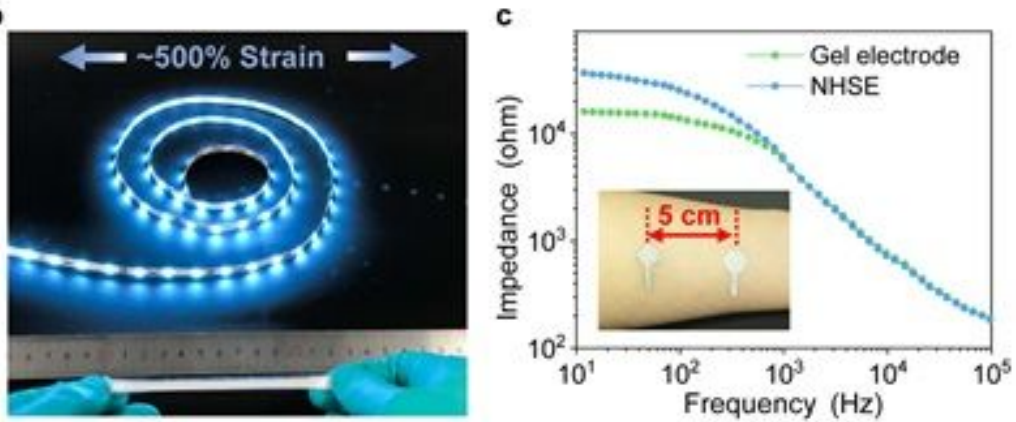

e

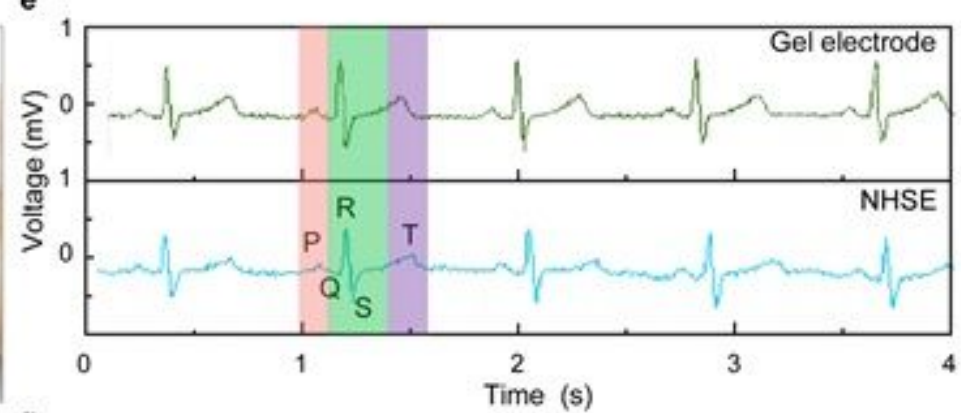

g

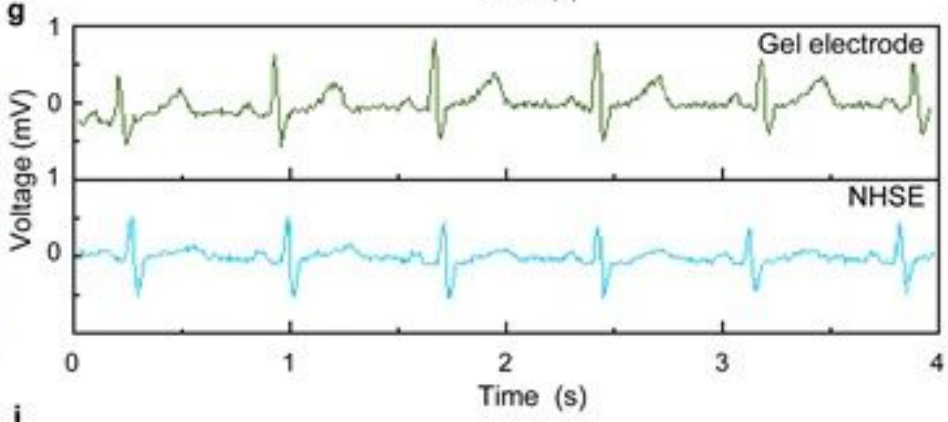

i

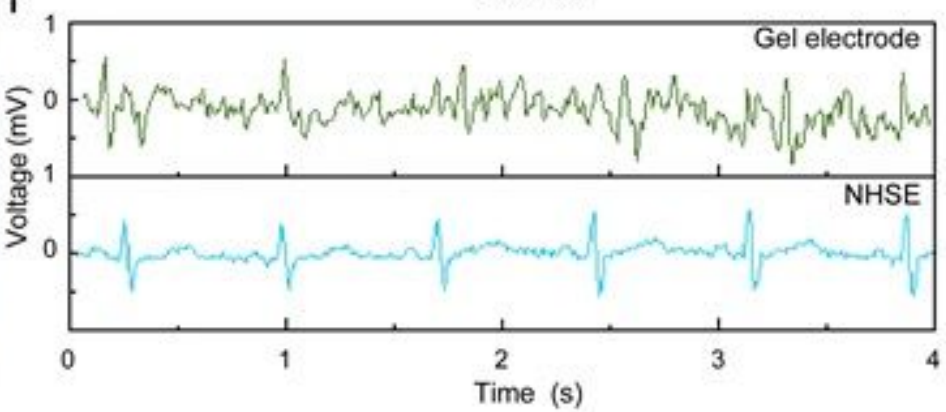

Figure 4

Application of the NHSE in elastic conductor and ECG monitoring. $a, b$ Photographs of the stretchable conductor to power a strip of LEDs under $0 \%$ strain (a) and 500\% strain (b). c Comparison of electrodeskin contact impedance of the NHSE and commercial gel electrode. $d$-i Schematics photographs of the NHSE for on-body healthcare monitoring and corresponding signals of commercial gel electrode (upper) and the NHSE (lower) while servicing in three scenes, i.e., working statically (d, e), doing exercise (f, g), and taking a shower $(h, i)$, respectively. 
a
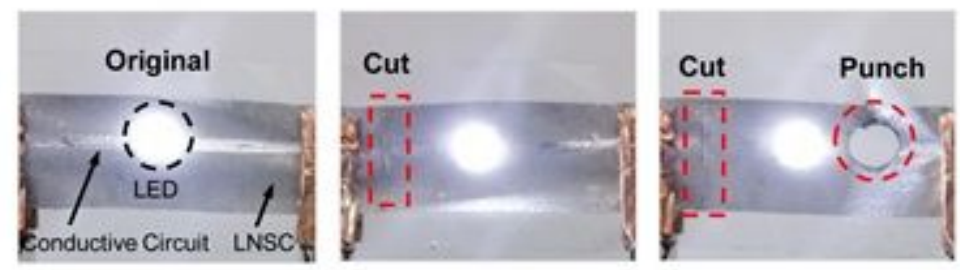

c

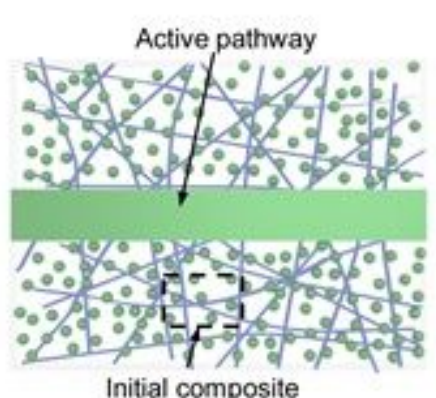

f

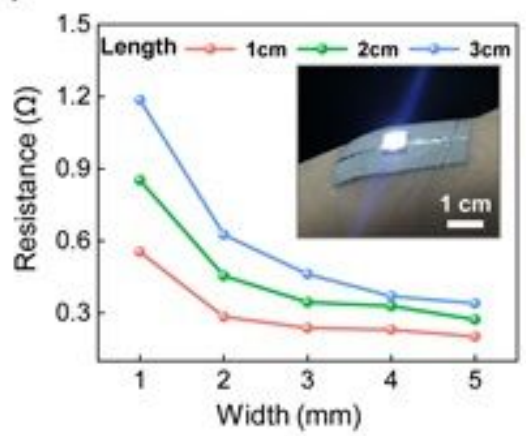

d

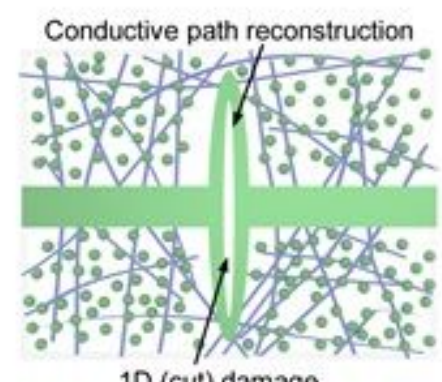

1D (cut) damage

g

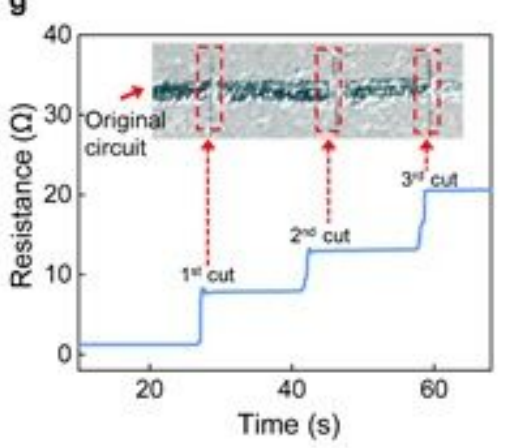

b
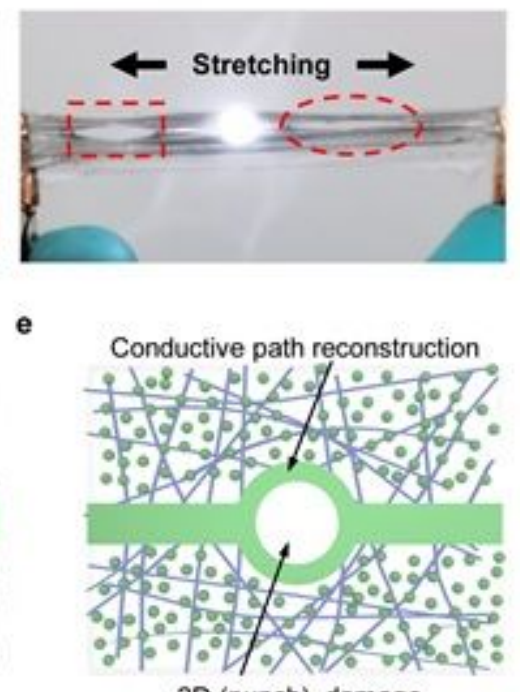

$2 \mathrm{D}$ (punch) damage

h

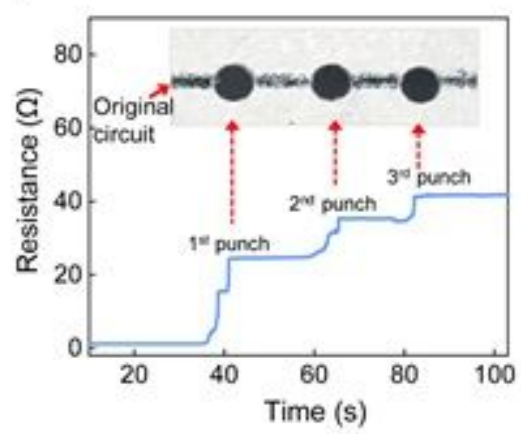

Figure 5

Electrically self-healing high-robust stretchable conductor. a Photographs of a selectively activated line on the LNSC connected with LED under original, cutting, hole punching status. b Photograph of the damaged circuit even upon stretching. c-e A schematic illustration of the electrically self-healing mechanism: (c) a conductive LM trace was selectively activated from initially insulating LM nanoparticle embedded in TPU nanofibers scaffold. (d, e) Following the damage, no matter for cut or hole punch, the conductive pathways autonomously reconstruct to continuously maintain the conductivity. $f$ the resistance of different length and width of the LM trace. Inset shows the photographs of LED connected by a conformable LM trance on an index finger knuckle. $g$, $h$ Resistance variation as a function of time under one-dimensional (cut) and two-dimensional (hole punch) damages.

\section{Supplementary Files}

This is a list of supplementary files associated with this preprint. Click to download.

- SupplementaryMaterialsforNC.docx

- SupplementaryMovie1.mp4

- SupplementaryMovie2.mp4 
- SupplementaryMovie3.mp4

- SupplementaryMovie4.mp4 